\title{
Soundscape expectations of rural tourism: A comparison between Chinese and English potential tourists
}

Xinxin Ren, Jian Kang, Peisheng Zhu, and Shiyuan Wang

Citation: The Journal of the Acoustical Society of America 143, 373 (2018); doi: 10.1121/1.5019466

View online: https://doi.org/10.1121/1.5019466

View Table of Contents: http://asa.scitation.org/toc/jas/143/1

Published by the Acoustical Society of America

\section{Articles you may be interested in}

Children's early bilingualism and musical training influence prosodic discrimination of sentences in an unknown language

The Journal of the Acoustical Society of America 143, EL1 (2018); 10.1121/1.5019700

Auditory stream segregation of iterated rippled noises by normal-hearing and hearing-impaired listeners

The Journal of the Acoustical Society of America 143, 378 (2018); 10.1121/1.5021333

Combining degradations: The effect of background noise on intelligibility of disordered speech

The Journal of the Acoustical Society of America 143, 281 (2018); 10.1121/1.5021254

Uncovering mental representations of smiled speech using reverse correlation

The Journal of the Acoustical Society of America 143, EL19 (2018); 10.1121/1.5020989

The impact of exploiting spectro-temporal context in computational speech segregation

The Journal of the Acoustical Society of America 143, 248 (2018); 10.1121/1.5020273

Pile driving acoustics made simple: Damped cylindrical spreading model

The Journal of the Acoustical Society of America 143, 310 (2018); 10.1121/1.5011158 


\title{
Soundscape expectations of rural tourism: A comparison between Chinese and English potential tourists
}

\author{
Xinxin Ren, ${ }^{1, a)}$ Jian Kang, ${ }^{2}$ Peisheng Zhu, ${ }^{1}$ and Shiyuan Wang ${ }^{1}$ \\ ${ }^{1}$ School of Architecture and Fine Art, Dalian University of Technology, Dalian 116024, People's Republic of \\ China \\ ${ }^{2}$ School of Architecture, The University of Sheffield, Sheffield S10 2TN, United Kingdom
}

(Received 18 May 2017; revised 25 October 2017; accepted 1 December 2017; published online 25 January 2018)

A questionnaire survey was carried out for a comparative study between Chinese and English potential tourists to examine their soundscape expectation. The results show that while both groups prefer natural sounds most, compared to the English, the Chinese expect natural, livestock, melodic sounds more, and traffic and industrial sounds less. The sound categories related to the interactions between human activities and nature play a more dominant role for the English than the Chinese, in terms of preference of sound sources. On the expectation of a holistic soundscape, function is the most important aspect for the Chinese, while that for the English is sound characteristics; the expected psychological perception for the English is associated with emotional response rather than basic ecology consciousness, as for the Chinese. (C) 2018 Acoustical Society of America. https://doi.org/10.1121/1.5019466

Pages: $373-377$

\section{INTRODUCTION}

The ISO 12913-1 defines soundscape as the perception and understanding of an acoustic environment, in context, by the individual, or by a society. ${ }^{1}$ One key factor in people's perception of a soundscape is the expectation of a context within cognition and emotion, which can also influence their behavior and evaluation of both a soundscape and location., ${ }^{2,3}$

The number of people visiting the countryside has increased with the recent rise in the demand for a simple and natural experience, especially in developing countries like China, where rural soundscapes are rather different from urban environments. This gives relief from cognitive overload and reduces stress. ${ }^{4,5}$ While many studies have been carried out on rural soundscapes, ${ }^{2-6}$ there is a lack of research on soundscape expectations that consider cultural differences.

In this study, therefore, a questionnaire survey-which is part of the larger research on soundscape design for urban residents-has been carried out to examine rural soundscape expectations in terms of the preference of sound sources and the expectation of holistic soundscapes, in particular, from the perspective of a cross-cultural comparison between Chinese and English people.

\section{METHODS}

The questionnaire survey was conducted for two groups of students: 153 Chinese students (who had been studying in the U.K. for less than two years), and 149 English students (aged $22 \pm 2 \mathrm{yr}$ ) who had been living in urban areas and had experiences of rural tourism in China and the UK, respectively, but did not frequently visit rural areas. The selection for this study was influenced by two reasons: (1) people who

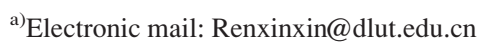

live in urban areas are potential rural tourists, whose expectations are significant considerations for facilitating rural soundscape design, and (2) as participants, young people tend to reflect on the prevailing norms, and their perception is more stable than that of other age groups in terms of the effect of experience and emotion, for example., ${ }^{2,4,7,8}$

The surveys were conducted in the libraries and in relatively quiet public rest spaces in the two universities of Sheffield (The University of Sheffield and Sheffield Hallam University). All the participants were volunteers. Prior to the survey, a short interview was conducted with randomly selected students and the survey was introduced. Then, participants that met the requirements of the survey (as listed in the previous paragraph) were asked to answer the questionnaire (the questionnaire was both in Chinese and English) to provide an evaluation of their own country's rural environment. At the beginning of the questionnaire, typical rural landscapes were mentioned to evoke the participants' prior experiences in the countryside. For example, in the English version of the questionnaire, typical villages located in Peak District National Park, a rural landscape dominated by heather moorlands and sheep farming ${ }^{8}$ that attracts a large number of visitors from urban areas, were presented; in the Chinese version of the questionnaire, a traditional rural landscape, centralized by human settlements and combined with the natural and agricultural landscape ${ }^{9}$ - a landscape that is well known by Chinese people-was presented. The evaluations were inquired from the viewpoint of rural tourists and not from the viewpoint of residents.

The questionnaire consisted of two aspects. (1) Evaluating the sound preferences from a series of sound sources. These were sounds frequently heard in English or Chinese rural environments, including sounds of traffic (motorbike, tractor working, road traffic, and bicycle), industrial sounds (construction and machinery), activity sounds 

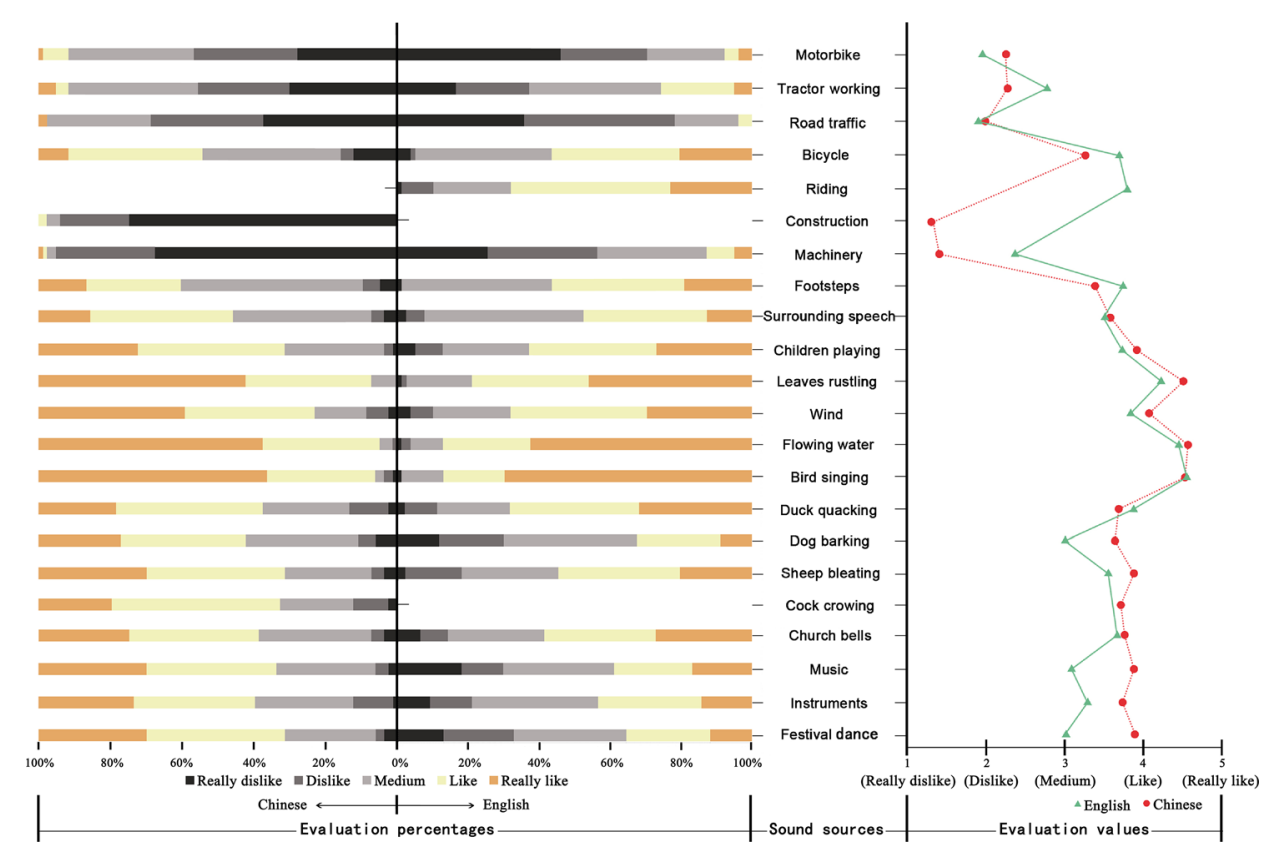

FIG. 1. (Color online) Preference of sound sources: A comparison between the Chinese and the English participants. (riding, footsteps, surrounding speech, and children playing), natural sounds (leaves rusting, wind, flowing water, and bird singing), livestock sounds (duck quacking, dog barking, sheep bleating, and cock crowing), and melodic sounds (church bells ringing, music, instruments, and festival dance). Corresponding to previous studies, ${ }^{7,10-12}$ a five-point Likert scale was used, as follows: (1) Really dislike; (2) Dislike; (3) Medium; (4) Like; and (5) Really like. (2) Evaluating the expectations from a holistic soundscape. The semantic differential method was applied to determine key factors that characterize the rural soundscape expectations. The participants were asked to provide their comments according to the following instructions: "Please provide your expectations of the aural perception of a rural acoustic environment according to the descriptions of 18 indices used, on a five-point bipolar rating scale." The indices were well understood and used for evaluation on soundscape and product sound quality in previous research. ${ }^{7,12-14}$

A database was then established in SPSS 17.0 (SPSS Statistics is a software package used for statistical analysis) for further analysis for reliability. The results demonstrated that the reliability coefficient alpha $=0.744-0.754>0.7$, which was acceptable. Simultaneously, the Kaiser-Meyer-Olkin (KMO) measure of sampling adequacy $=0.656 \sim 0.738$ and the corresponding Bartlett's spherical test results ( $p=0.000>0.01)$ were also found to satisfy the validity of the questionnaire $(0.6<\mathrm{KMO}<0.7) .^{10,15,16}$

\section{RESULTS}

\section{A. Preference of sound sources}

Corresponding to the results of earlier studies, the potential tourists demonstrated a positive attitude towards natural sounds. From the percentage of sound preferences, as shown in Fig. 1, it is clear that over $68 \%$ of the participants chose "like" and "really like" for natural sounds; within this, even more Chinese reacted favorably (by $10 \%$ on average) to the sounds of leaves rustling, wind, flowing water, and bird singing. Industrial sounds, such as those of construction and machinery, however, were the least favorite sounds for most Chinese as up to $90 \%$ of the evaluations were "dislike" and "really dislike." The sounds of construction are not frequently heard in English rural areas and were not evaluated by more than $50 \%$ of the English participants (it was the same for the other missing sound sources); however, most English people dislike the sound of traffic, with more than $65 \%$ of evaluations being below "medium" for motorbikes and road traffic sounds. Using a paired-samples t-test, the scores of the sound preferences between the Chinese and English were examined. For certain sound sources, including tractor working, bicycle, machinery, footsteps, leaves rustling, dog barking, music, instruments, and festival dance, there were significant differences $(p<0.05)$ between the Chinese and English. More specifically, from the mean values of sound preferences shown in Fig. 1, it is evident that compared to the English, the Chinese prefer natural, livestock, and melodic sounds more (by $0.12,0.20$, and 0.54 on average, respectively) and expected traffic and industrial sounds less (with evaluation scores lower by about 0.30 , on average).

To reveal the main sound categories that affect soundscape expectations, a factor analysis was done using the data of Chinese sound preferences. The varimax rotated principal component analysis was employed to extract the orthogonal. With a criterion factor of eigenvalue $>1$, six factors were determined, as shown in Table I. Factor 1 (18.59\%) is mainly associated with livestock and natural sounds; factor 2 $(12.64 \%)$ is generally associated with traffic and industrial sounds; factor $3(11.75 \%)$ is mostly associated with melodic sounds. Correspondingly, from the sound categories based on a factor analysis of English data, it is interesting to note that, different from the Chinese results, factor $1(16.77 \%)$ which includes flowing water, surrounding speech, bird singing and footsteps, principally relates to an atmosphere of 
TABLE I. Factor analysis of sound preference: overall results of the Chinese/English participants. Kaiser-Meyer-Olkin measure of sampling adequacy: 0.738/0.656 ( $p=0.000)$; cumulative \%: 69.62/73.39; extraction method: principal component analysis; rotation method: Varimax with Kaiser normalization; $\mathrm{N}=153 / 149$.

\begin{tabular}{|c|c|c|c|c|c|c|c|}
\hline \multirow[b]{2}{*}{ Sounds } & \multicolumn{7}{|c|}{ Factors (the numbers in the brackets are explained variance for Chinese/English) } \\
\hline & $\begin{array}{c}1 \\
(18.59 / 16.77 \%)\end{array}$ & $\begin{array}{c}2 \\
(12.64 / 13.12 \%)\end{array}$ & $\begin{array}{c}3 \\
(11.75 / 10.15 \%)\end{array}$ & $\begin{array}{c}4 \\
(11.36 / 8.71 \%)\end{array}$ & $\begin{array}{c}5 \\
(9.17 / 8.47 \%)\end{array}$ & $\begin{array}{c}6 \\
(6.11 / 8.22 \%)\end{array}$ & $\begin{array}{c}7 \\
(-/ 7.95 \%)\end{array}$ \\
\hline Duck quacking & $\mathbf{0 . 8 2} / 0.22$ & $0.02 / 0.03$ & $-0.10 /-0.22$ & $0.01 / 0.44$ & $-0.34 / 0.55$ & $-0.19 / 0.02$ & $-/ 0.41$ \\
\hline Cock crowing & $0.81 /-$ & $-0.08 /-$ & $-0.20 /-$ & $0.08 /-$ & $-0.34 /-$ & $-0.18 /-$ & - \\
\hline Dog barking & $\mathbf{0 . 7 6} / 0.35$ & $-0.01 / 0.41$ & $-0.32 / 0.03$ & $0.23 /-0.40$ & $-0.09 / 0.23$ & $-0.17 / 0.16$ & $-/ 0.15$ \\
\hline Sheep bleating & $\mathbf{0 . 7 5} / 0.43$ & $-0.01 / 0.13$ & $-0.26 / 0.06$ & $0.15 /-0.08$ & $-0.23 / 0.71$ & $-0.24 / 0.33$ & $-/ 0.05$ \\
\hline Children playing & $\mathbf{0 . 6 7 / 0 . 4 6}$ & $0.08 / 0.41$ & $0.19 / 0.27$ & $-0.43 / 0.16$ & $0.21 / 0.21$ & $0.12 / 0.42$ & $-/-0.24$ \\
\hline Wind & $\mathbf{0 . 6 5} / 0.50$ & $-0.05 /-0.10$ & $-0.04 / \mathbf{0 . 5 7}$ & $-0.38 /-0.01$ & $0.04 / 0.07$ & $0.24 /-0.17$ & $-/ 0.36$ \\
\hline Flowing water & $0.65 / 0.82$ & $-0.34 /-0.04$ & $-0.02 / 0.11$ & $0.11 /-0.10$ & $0.41 /-0.07$ & $0.00 / 0.08$ & $-/ 0.05$ \\
\hline Leaves rustling & $\mathbf{0 . 6 3} / 0.17$ & $-0.18 / 0.13$ & $-0.25 /-0.07$ & $-0.06 / 0.03$ & $0.18 / 0.04$ & $0.28 /-0.04$ & $-/ 0.90$ \\
\hline Bird singing & $0.56 / 0.81$ & $-0.49 / 0.01$ & $-0.23 / 0.01$ & $0.04 /-0.13$ & $0.21 / 0.29$ & $-0.09 /-0.01$ & $-/ 0.16$ \\
\hline Motorbike & $0.32 /-0.05$ & $0.64 / 0.24$ & $0.24 /-0.14$ & $0.28 / 0.19$ & $0.26 /-0.70$ & $0.10 / 0.29$ & $-/ 0.08$ \\
\hline Construction & $0.18 /-$ & $0.60 /-$ & $0.26 /-$ & $-0.01 /-$ & $-0.34 /-$ & $0.08 /-$ & - \\
\hline Tractor working & $0.54 / 0.14$ & $\mathbf{0 . 5 9 / 0 . 0 9}$ & $-0.04 / 0.10$ & $0.29 / 0.07$ & $0.24 /-0.07$ & $0.20 / 0.57$ & $-/ 0.51$ \\
\hline $\begin{array}{l}\text { Music (music from public } \\
\text { broadcast/street music) }\end{array}$ & $0.24 / 0.31$ & $-0.57 / 0.55$ & $0.40 /-0.20$ & $0.38 / 0.45$ & $0.03 /-0.19$ & $0.00 /-0.05$ & $-/-0.01$ \\
\hline Machinery & $0.20 /-0.07$ & $\mathbf{0 . 4 9} / 0.17$ & $0.35 / 0.00$ & $-0.09 / 0.83$ & $-0.44 /-0.11$ & $-0.06 / 0.08$ & $-/ 0.07$ \\
\hline Road traffic & $0.35 /-0.07$ & $\mathbf{0 . 4 8} /-0.19$ & $-0.08 /-0.03$ & $0.28 / 0.04$ & $-0.06 /-0.01$ & $0.45 / 0.89$ & $-/-0.08$ \\
\hline Church bells & $0.34 / 0.11$ & $-0.43 / \mathbf{0 . 6 0}$ & $0.38 /-0.42$ & $-0.23 / 0.14$ & $-0.21 / 0.17$ & $0.15 /-0.23$ & $-/ 0.16$ \\
\hline Bicycle & $0.17 / 0.17$ & $0.42 / 0.13$ & $0.25 / \mathbf{0 . 8 5}$ & $0.38 /-0.09$ & $0.35 /-0.03$ & $-0.37 /-0.02$ & $-/-0.12$ \\
\hline $\begin{array}{l}\text { Instruments (instruments/ } \\
\text { brass band music) }\end{array}$ & $0.16 /-0.05$ & $-0.48 / \mathbf{0 . 8 2}$ & $0.61 / 0.82$ & $0.33 / 0.07$ & $-0.01 /-0.13$ & $0.04 /-0.02$ & $-/ 0.14$ \\
\hline $\begin{array}{l}\text { Festival dance (yangko/ } \\
\text { morris dance) }\end{array}$ & $0.47 /-0.14$ & $-0.31 / \mathbf{0 . 8 7}$ & $\mathbf{0 . 4 9} / 0.10$ & $0.04 / 0.00$ & $-0.13 /-0.01$ & $0.18 /-0.03$ & $-/-0.03$ \\
\hline Surrounding speech & $0.45 / \mathbf{0 . 8 1}$ & $0.25 /-0.04$ & $0.10 /-0.02$ & $-0.53 / 0.27$ & $0.20 / 0.11$ & $0.07 /-0.10$ & $-/ 0.17$ \\
\hline Footsteps & $0.27 / \mathbf{0 . 5 8}$ & $0.26 / 0.11$ & $0.35 / 0.44$ & $-0.41 /-0.15$ & $0.24 / 0.24$ & $-0.56 / 0.06$ & $-/-0.03$ \\
\hline Riding & $-/-0.08$ & $-/-0.02$ & $-/ 0.62$ & $-/ 0.52$ & $-/ 0.18$ & $-/ 0.17$ & $-/ 0.03$ \\
\hline
\end{tabular}

natural environment and activities. Factor $2(13.12 \%)$ mainly contains melodic sounds; factor $3(10.15 \%)$ indicates that people are more concerned about the aural perception of field activities, which include bicycle, riding, wind, etc. In other words, beside natural and melodic sounds, the sound categories related to the interactions between human activities and nature played the most important role for the English, whereas livestock, traffic, and industrial sounds were the dominant sounds that influenced Chinese soundscape expectations. ${ }^{17}$

\section{B. Expectation of a holistic soundscape}

In terms of the aural perception of holistic soundscapes, the expectations of the Chinese and the English were generally similar, as can be seen from Fig. 2, which shows the results of Chinese and English evaluations covering various aspects including satisfaction, strength, fluctuation, and social aspect. ${ }^{7,13}$ Although people from the two different cultures have similar tendencies in their expectations, a notable difference occurs for simple-varied relating to the fluctuation in the soundscape, suggesting that the aural perception of "varied" is perhaps more expected by the English.

To explore the characterization of soundscape expectations, the evaluations of holistic soundscapes are further analyzed through factor analysis, as shown in Table II. For the Chinese, it can been seen that factor $1(17.20 \%)$ is related to the function of a relaxing experience, including artificial-natural, noisy-quiet, unpleasant-pleasant, harsh-gentle, dislike-like, and boringinteresting. Factor $2(12.99 \%)$ is more associated with the sound characteristics, including sharp-flat, slow-fast, hard-soft, rough-smooth, and deadly-echoed. Factor 3 $(12.03 \%)$ is concerned with psychological perception, including the ecological consciousness of agitating-calming and uncomfortable-comfortable. Factor $4(8.92 \%)$ is mainly related to communication. Factor $5(8.83 \%)$ tends to connect with spatiality, including everywhere-directional and close-far. For the results of the English, factor 1 $(15.33 \%)$ is related to the sound characteristics. Factor 2 mainly focuses on relaxation. Factor 3 (13.69\%) includes the perception of boring-interesting, unsocial-social, and meaningless-meaningful. Factor $4(9.42 \%)$ and factor 5 $(7.10 \%)$ are related to spatiality.

In general, the factors cover the main aspects of designing the acoustics of a rural soundscape-function, sound characteristics, psychological perception, and space-and include elements of sound, users, and environment. However, the most significant aspect of rural soundscape expectations is function (relaxation) for the Chinese, whereas for the English, it is sound characteristics. In terms of psychological perception, instead of the basic need of "calming" and "comfortable" expected by the Chinese, the English look forward to a varied soundscape that is 


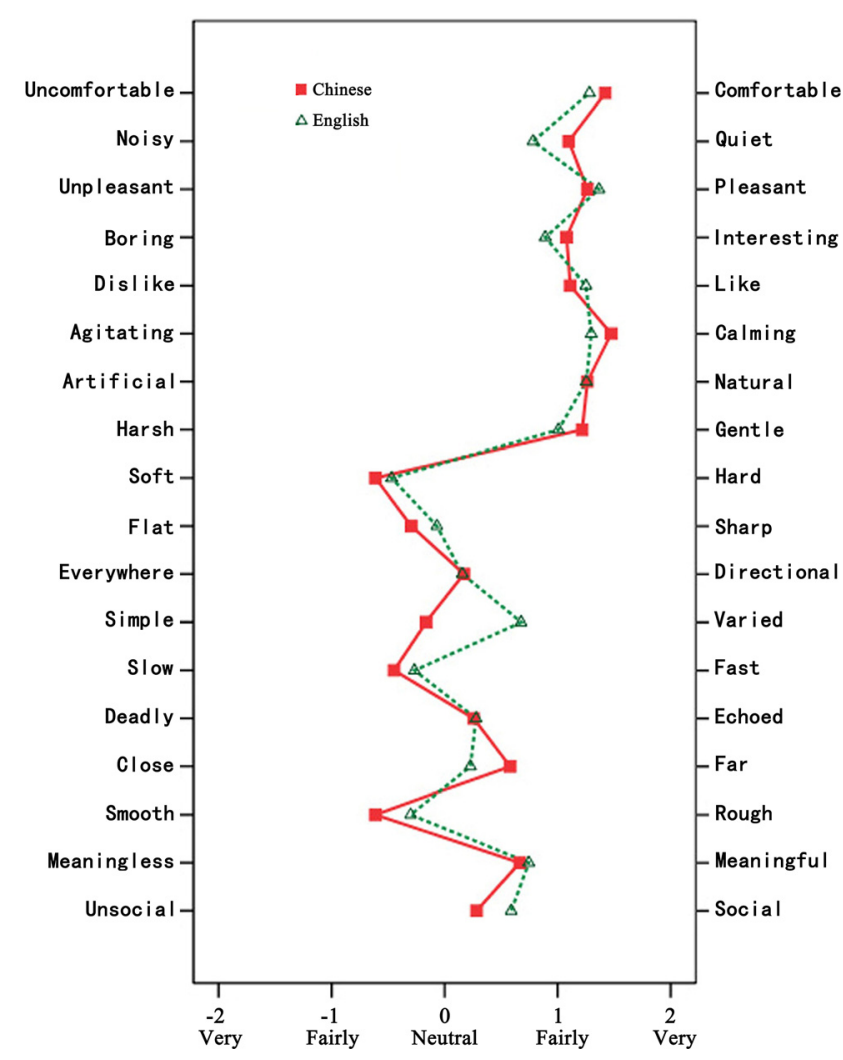

FIG. 2. (Color online) Expectation of a holistic soundscape: A comparison between the Chinese and the English participants.

"interesting," "social," and "meaningful," to satisfy an emotional response. Space, the last factor for the Chinese and English, both, was not as important as the other aspects, which suggests that the relationship between sound and the users may be of greater significance than that between sound and the environment in a holistic soundscape.

\section{DISCUSSION}

Previous studies have suggested that the sound preferences of older people tend to be shaped by experience, and that they may be more appreciative of natural and culturally approved sounds, with more emotion, whereas teenagers $\left(10-17 \mathrm{yr}\right.$ old) may prefer a high-arousal soundscape. ${ }^{7}$ Simultaneously, the expectations of a soundscape are based on prior experience when perceiving soundscape contexts. ${ }^{2}$ Overall, the results from this study support the theory that belonging to a particular culture (English-Chinese based) influences the evaluation of a soundscape. However, with regard to how potential visitors might shape their preferences and expectations of a rural soundscape depending on culture differences, this study mainly focuses on young people with fewer experiences of visiting rural areas. In terms of the expectations of a holistic soundscape, it is noted that the five factors occupied $59.85 \%-59.97 \%$ of the total variance, corresponding to $53 \%$ in urban soundscape evaluations with the same adjectives. ${ }^{7,13}$ It is therefore suggested that connotative and denotative meanings of the indices are available for rural soundscape expectations. For the $40 \%$ uncovered variability for both the Chinese and the English, the reason is perhaps due to the significant variations within the contextual domain ${ }^{18}$ of rural areas and the characteristics of the sound sources. Another possible reason is that the indices that are probably well fitted to a rural soundscape in terms of history, culture, and human health, have not been explored. To make a more concrete statement about these aspects, further research is necessary.

TABLE II. Factor analysis of expectations of a holistic soundscape: overall results of the Chinese/English participants. Kaiser-Meyer-Olkin measure of sampling adequacy: 0.720/0.683 $(p=0.000)$; cumulative \%: 59.97/59.85; extraction method: principal component analysis; rotation method: Varimax with Kaiser normalization; $\mathrm{N}=153 / 149$.

\begin{tabular}{|c|c|c|c|c|c|}
\hline \multirow[b]{2}{*}{ Indices } & \multicolumn{5}{|c|}{ Factors (the numbers in the brackets are explained variance for Chinese/English) } \\
\hline & $\begin{array}{c}1 \\
(17.20 / 15.33 \%)\end{array}$ & $\begin{array}{c}2 \\
(12.99 / 14.31 \%)\end{array}$ & $\begin{array}{c}3 \\
(12.03 / 13.69 \%)\end{array}$ & $\begin{array}{c}4 \\
(8.92 / 9.42 \%)\end{array}$ & $\begin{array}{c}5 \\
(8.83 / 7.10 \%)\end{array}$ \\
\hline Artificial-natural & $\mathbf{0 . 8 0} /-0.30$ & $-0.02 / \mathbf{0 . 6 1}$ & $0.04 / 0.18$ & $-0.08 / 0.11$ & $0.05 /-0.17$ \\
\hline Noisy-quiet & $0.78 / 0.12$ & $-0.13 / 0.68$ & $0.04 / 0.09$ & $-0.10 / 0.06$ & $0.07 /-0.09$ \\
\hline Unpleasant-pleasant & $\mathbf{0 . 7 2} /-0.33$ & $-0.19 / 0.57$ & $0.29 / 0.46$ & $-0.09 /-0.03$ & $0.21 /-0.12$ \\
\hline Harsh-gentle & $0.59 /-0.55$ & $-0.33 / 0.37$ & $0.27 / 0.12$ & $-0.02 / 0.35$ & $-0.08 /-0.19$ \\
\hline Dislike-like & $\mathbf{0 . 5 6} /-0.37$ & $0.02 / 0.29$ & $0.48 / 0.47$ & $0.13 / 0.26$ & $0.31 / 0.02$ \\
\hline Boring-interesting & $\mathbf{0 . 4 9} /-0.05$ & $-0.07 / 0.03$ & $0.47 / \mathbf{0 . 8 0}$ & $0.32 / 0.07$ & $0.28 /-0.15$ \\
\hline Sharp-flat & $-0.05 / \mathbf{0 . 7 5}$ & $\mathbf{0 . 7 1} / 0.29$ & $0.00 / 0.00$ & $0.21 / 0.13$ & $-0.17 / 0.07$ \\
\hline Slow-fast & $-0.08 / 0.39$ & $0.64 /-0.11$ & $-0.16 / 0.15$ & $-0.09 /-0.11$ & $-0.01 / \mathbf{0 . 7 2}$ \\
\hline Hard-soft & $-0.26 / \mathbf{0 . 7 6}$ & $\mathbf{0 . 6 4} /-0.26$ & $-0.10 / 0.08$ & $0.11 / 0.07$ & $0.10 / 0.07$ \\
\hline Rough-smooth & $-0.32 / \mathbf{0 . 7 3}$ & $\mathbf{0 . 5 8} /-0.23$ & $-0.03 /-0.14$ & $0.41 / 0.17$ & $-0.13 /-0.20$ \\
\hline Deadly-echoed & $0.32 / 0.18$ & $\mathbf{0 . 5 5} / 0.17$ & $-0.11 / 0.17$ & $-0.42 / \mathbf{0 . 6 0}$ & $0.27 / 0.16$ \\
\hline Agitating-calming & $0.16 /-0.34$ & $-0.24 / 0.38$ & $\mathbf{0 . 8 0} / 0.05$ & $-0.20 / 0.56$ & $0.01 /-0.07$ \\
\hline Uncomfortable-comfortable & $0.23 /-0.22$ & $-0.13 / \mathbf{0 . 6 3}$ & $\mathbf{0 . 8 0} / 0.14$ & $-0.13 / 0.36$ & $0.10 / 0.19$ \\
\hline Unsocial-social & $0.04 / 0.10$ & $-0.02 / 0.01$ & $-0.17 / \mathbf{0 . 7 5}$ & $\mathbf{0 . 7 8} /-0.13$ & $0.04 / 0.10$ \\
\hline Simple-varied & $-0.14 / 0.08$ & $0.31 /-\mathbf{0 . 6 0}$ & $-0.03 / 0.45$ & $\mathbf{0 . 5 8} / 0.16$ & $-0.11 /-0.08$ \\
\hline Meaningless-meaningful & $0.25 /-0.06$ & $0.02 / 0.16$ & $0.21 / \mathbf{0 . 6 6}$ & $0.16 / 0.23$ & $\mathbf{0 . 7 4} / 0.03$ \\
\hline Everywhere-directional & $0.07 / 0.16$ & $-0.15 /-0.11$ & $-0.34 / 0.00$ & $-0.08 / \mathbf{0 . 6 9}$ & $\mathbf{0 . 5 8} / 0.00$ \\
\hline Close-far & $-0.01 /-0.24$ & $0.03 /-0.04$ & $0.18 /-0.18$ & $-0.14 / 0.23$ & $0.58 / 0.72$ \\
\hline
\end{tabular}




\section{CONCLUSIONS}

The results of this cross-cultural comparison demonstrate that while both groups prefer natural sounds the most, compared to the English, the Chinese expect more natural, livestock, and melodic sounds and less traffic and industrial sounds. Beside the natural and melodic sounds, the sound categories related to the interactions between human activities and nature play the most important role for the English, whereas livestock, traffic, and industrial sounds are the dominant sounds that influence Chinese expectations of sound sources.

In terms of the expectations of a holistic soundscape, the function, sound characteristics, and psychological perception are significant aspects for both groups. Function is the most important for the Chinese, while for the English, sound characteristics are the most important. The expected psychological perception for the English is associated with an emotional response, such as "interesting," "social," and "meaningful," rather than with the basic ecological consciousness, as for the Chinese, such as "calming" and "comfortable."

\section{ACKNOWLEDGMENTS}

The authors are indebted to Francesco Aletta and Fangxin Zhao for helping with the questionnaire survey in Sheffield. The authors also wish to specially thank the participants who participated in the present study. The research was supported by the European project "Urban Sound Planner," the Natural Science Foundation of China (51741803), the Fundamental Research Funds for the Central Universities (DUT17RC(3)018), the Social Science Association Fund of Liaoning Province (20181slktyb-023), and the NSFC (51778100).

${ }^{1}$ ISO 12913-1:2014, “Acoustics. Soundscape. Part 1: Definition and conceptual framework" (International Organization for Standardization, Geneva, Switzerland, 2014).
${ }^{2}$ N. S. Bruce and W. J. Davies, "The effects of expectation on the perception of soundscapes," Appl. Acoust. 85, 1-11 (2014).

${ }^{3}$ D. Botteldooren and B. D. Coensel, "Quality labels for the quiet rural soundscapes," in Proceedings of Internoise, Honolulu, HI (2006).

${ }^{4} \mathrm{X}$. Ren and J. Kang, "Interactions between landscape elements and tranquility evaluation based on eye tracking experiments," J. Acoust. Soc. Am. 138(5), 3019-3022 (2015).

${ }^{5}$ G. R. Watts and R. J. Pheasant, "Factors affecting tranquility in the countryside," Appl. Acoust. 74, 1094-1103 (2013).

${ }^{6}$ K. C. Lam, A. L. Brown, L. Marafa, and K. C. Chau, "Human preference for countryside soundscapes," Acta Acust. united Acust. 96, 463-471 (2010).

${ }^{7}$ J. Kang, Urban Sound Environment—Chapter Three: Urban Soundscape (Taylor \& Francis, London/Spon, London, 2006).

${ }^{8}$ N. Suckall, E. D. G. Fraser, T. Cooper, and C. Quinn, "Visitor perceptions of rural landscape: A case study in the Peak District National Park, England,” J. Environ Manage. 90, 1195-1203 (2010).

${ }^{9}$ Y. C. Wang and B. Y. Liu, "Discussions on rural landscape and rural landscape planning in China," Chin. Landscape Archit. 5, 76-79 (2003) (in Chinese).

${ }^{10}$ E. Rogge, F. Nevens, and H. Gulinck, "Perception of rural landscapes in Flankers: Looking beyond aesthetics," Landscape Urban Plann. 82, 159-174 (2007).

${ }^{11}$ X. Ren and J. Kang, "Effects of the visual landscape factors of an ecological waterscape on acoustic comfort," Appl. Acoust. 96, 171-179 (2015).

${ }^{12} \mathrm{X}$. Ren and J. Kang, "Comparative study on sound preferences between Chinese and English rural tourists," in Proceedings of the 24th International Congress on Sound and Vibration, London, United Kingdom, 2017.

${ }^{13}$ J. Kang and M. Zhang, "Semantic differential analysis of the soundscape in urban open public Spaces," Build. Environ. 45, 150-157 (2010).

${ }^{14}$ B. Schulte-Fortkamp, "The quality of acoustic environments of acoustic environments and the meaning of soundscapes," in Proceedings of the 17th International Congress on Acoustics, Rome, Italy, 2001.

${ }^{15}$ F. J. Palmer and R. E. Hoffman, "Rating reliability and representation validity in scenic landscape assessment," Landscape Urban Plann. 54, 149-161 (2001).

${ }^{16} \mathrm{M}$. Topaloglu, E. Caldibi, and G. Oge, "The scale for the individual and social impact of students' social network use: The validity and reliability studies," Comput. Human Behav. 61, 350-356 (2016).

${ }^{17} \mathrm{X}$. Ren and J. Kang, "Comparative study on sound preferences between Chinese and English rural tourists," Urban. Archit. 10, 114-117 (2016) (in Chinese).

${ }^{18}$ D. A. Hall, A. Irwin, M. Edmondson-Jones, S. Phillips, and J. E. W. Poxon, "An exploratory evaluation of perceptual, psychoacoustic and acoustical properties of urban soundscapes," Appl. Acoust. 74, 248-254 (2013). 